\title{
Caries oclusal incipiente : Un nuevo enfoque
}

Nishiyama Machado de Almeida C, Geller Palti D, Francisconi Silveira PA. Caries oclusal incipiente : Un nuevo enfoque. Rev Estomatol Herediana. 2006; 16 (2) : 126 - 130.

\section{RESUMEN}

No obstante que el conocimiento sobre el diagnóstico y tratamiento de la caries dental ha avanzado, el diagnóstico de las lesiones oclusales todavía constituye un reto para los clínicos y los epidemiólogos. Los conocimientos sobre la enfermedad, así como los conceptos, formas de diagnóstico y tratamiento de la odontología han evolucionado debido, principalmente, a tres grandes factores: (1) la visión de la caries dental como una enfermedad y, consecuentemente, la posibilidad de intervenir más tempranamente; (2) el uso del flúor y (3) la aparición de materiales restauradores adhesivos, que permiten restauraciones más conservadoras. Esta nueva perspectiva ha conducido al surgimiento de una odontología preventiva que hoy reconoce la presencia de lesiones cariosas ocultas, menores e incipientes. La necesidad del diagnóstico temprano es fundamental, en el sentido de que mientras más pronto se realice la intervención, menor será la pérdida de estructura dentaria y mayor la posibilidad de éxito en la recuperación de la salud bucal. En los últimos tiempos han surgido diversos métodos de diagnóstico y, con ellos, dudas sobre cuál es el ideal y que pueda, además, cumplir las siguientes características: alto grado de sensibilidad y especificidad, bajo costo y fácil aplicación diaria. A pesar de los grandes adelantos tecnológicos observados en el diagnóstico de caries dental, se considera que el examen clínico correcto, acompañado por el examen radiográfico, es un método eficaz para el diagnóstico de lesiones incipientes, que se caracterizan por aparecer principalmente en las superficies oclusales. Cuando hay dudas sobre el diagnóstico de lesiones incipientes se recomiendan métodos alternos y complementarios de tipo invasivo.

Palabras clave: CARIES DENTAL. diagnóstico.

A new look: incipient occlusal caries

ABSTRACT

Even though the knowledge about diagnosis and treatment of dental caries is well established, occlusal caries diagnosis remains a great challenge for clinicians and epidemiologists. New concepts of dental caries as a disease and the possibility of an early treatment as well as the use of fluoride and development of adhesive materials, which permit more constructive restorative procedures may be responsible for a new perception of a preventive dentistry. Allowing the appearance of new type of lesions such as hidden caries, incipient caries and smaller caries. For these reasons, the need of an early diagnosis becomes fundamental, because of the aim of modern dentistry as a preventive approach rather than invasive repair of the disease. In the last years, new diagnosis methods were implemented bringing new doubts about which shows the accurate result. The ideal diagnosis method should have high sensibility and specificity, low cost and should be easy to work. Even with the great technologies in new diagnosis tools, an accurate clinical and radiographic exam are considered an effective method in the diagnosis of incipient caries lesions. These incipient caries lesions mostly appear in the occlusal surfaces. In case of doubtful fissure caries an invasive diagnosis is established.

Key words: DENTAL CARIES. diagnosis.
Cristiane Nishiyama Machado de Almeida ${ }^{1}$

\section{Dafna Geller Palti ${ }^{2}$}

Paulo A. Francisconi Silveira ${ }^{3}$

Master en Dentística, área de concentración Materiales Dentales

Master en Odontopediatria

${ }^{3}$ Docente del Departamento de Dentística, Endodoncia

y Materiales Dentales

y Macultad de Odontología de Bauru - Universidad de Fãultad de Odont

\section{Correspondencia}

Cristiane Nishiyama Machado de Almeid Av. Dr. Octavio Pinheiro Brisolla 975

Vila Cidade Universitária CEP-17012101

Bauru, São Paulo, Brasil

Teléfono: 1432358267

e-mail: crisman@terra.com.br

Recibido : 14 de setiembre del 2006

Aceptado : 7 de marzo del 2007
A inicio del siglo XIX, dos grandes personajes establecieron los conceptos modernos sobre la caries dental. El primero fue Miller, quien sugirió en su trabajo titulado "Los microorganismos de la boca humana", que las bacterias serían responsables de la aparición de la caries (1). Por su parte, Black, describió de forma precisa que "...el inicio de la caries dental ocurre en los puntos en los que se favorece el alojamiento o agregación de microorganismos, los cuales no podrán estar sujetos a la remoción mecánica frecuente para prevenir su crecimiento continuo y acentuado." Además, Black fue el primero en describir el "biofilm" bacteriano, usando el término "placas gelatinosas" (1). Apartir de estos fundamentos, el concepto de la caries como enfermedad ha evolucionado y, al mismo tiempo, han ocurrido cambios en su prevalencia, así como en los métodos de diagnóstico y tratamiento, pudiéndose observar, principalmente en los últimos años, la valorización de una filosofía más preventiva y conservadora.

De estos estudios se obtuvieron tres resultados principales que produjeron un enorme impacto y modificaron algunos conceptos de la odontología restauradora y la cariología de entonces. Estos son: el enfoque del papel de la nutrición y de los azúcares en relación a la experiencia de caries; la utilización del flúor como método sistémico, responsable de la reducción significativa de la severidad y el número de lesiones cariosas, principalmente en niños (2-4) y la técnica de acondicionamiento ácido del esmalte, propuesta por Buonocore (5) en 1955, que sirvió de base para la utilización de materiales restauradores adhesivos y, consecuentemente, el surgimiento de una odontología restauradora más conservadora. 
La integración de estos conceptos ha conducido a la visión actual de la caries dental como enfermedad, que puede ser definida como una entidad multifactorial, crónica, infecciosa, de evolución rápida o lenta, que afecta las superficies lisas y las regiones de fosas y fisuras, pudiendo sus lesiones ser controladas y revertidas en los estadios iniciales.

Sin embargo, frente a los conocimientos actuales de esta enfermedad, al uso universal del flúor, a la mayor concienciación de profesionales y pacientes y a la reducción de su prevalencia y gravedad en los últimos años, la caries dental debe ser considerada aún un problema de salud pública en muchos países $(4,6)$, por lo que es necesario darle una atención constante, principalmente en la superficie oclusal, para diagnosticarla y tratarla de forma adecuada y lo más tempranamente posible $(7,8)$.

Meiers y Jensen relacionaron la reducción de la prevalencia y el patrón de caries dental con una mayor dificultad para diagnosticarla, por las dudas que se generan en el clínico sobre la necesidad de intervenir o no, especialmente en los casos de lesiones incipientes en las regiones de fosas y fisuras, siendo necesario contar con métodos de diagnóstico más precisos y objetivos (4).

Junto a estos factores, la práctica de una odontología con una filosofía más preventiva ha llevado también a la aparición de un nuevo perfil de pacientes: libres de caries, con lesiones de progresión más lenta y con lesiones incipientes, con una modalidad de diferente desarrollo, denominada caries oculta.

De los distintos tipos de lesiones cariosas, las lesiones de fosas y fisuras afectan al $80 \%$ de la población joven (9). En función de este grado de prevalencia, todavía alto, y de la dificultad para hacer un diagnóstico preciso, es que hoy se da mayor importancia a un diagnóstico y tratamiento tempranos. Por este motivo, el objetivo de este trabajo es ofrecer al cirujano-dentista clínico una visión actual de las particularidades de la caries dental incipiente en la superficie oclusal y abordar, también, los diferentes medios de diagnóstico disponibles y su efectividad.

\section{Caries oclusal}

La caries dental es un proceso dinámico, resultado de un desequilibrio en el mecanismo de desmineralización y remineralización que ocurre continuamente y de manera natural en la cavidad bucal, que se manifiesta cuando predomina la salida de iones de calcio y fosfato y, consecuentemente, causan la pérdida de minerales de la estructura dentaria. Inicialmente, esta pérdida ocurre a nivel ultraestructural pero, si no es controlada, puede llevar a la destrucción del diente.

El primer signo visible de la enfermedad es la aparición de la denominada "mancha blanca", que clínicamente se observa en la superficie dental seca como un esmalte poroso y de apariencia opaca y, al microscopio, como un aumento de los espacios intercristalinos. También se le conoce como lesión cariosa incipiente. En esta etapa de la enfermedad, la lesión tiene carácter reversible, siendo fundamental un diagnóstico precoz, preciso y sencillo.

Las superficies oclusales son más fácilmente afectadas por la caries dental, ya que presentan áreas estrechas, inaccesibles a la limpieza mecánica (1). Se considera que el estadio de erupción, la función de los dientes y la anatomía dental son factores importantes que favorecen la acumulación bacteriana y el inicio del desarrollo de lesiones en este tipo de superficie (10-12).

El período de erupción es una fase crítica para la formación de la caries dental. En esta etapa ocurren innumerables procesos microscópicos de disolución y reposición de minerales en la interfase esmaltebiofilm. Los dientes recién erupcionados presentan un esmalte parcialmente mineralizado, ya que todavía no han pasado por la llamada maduración posteruptiva y, por lo tanto, son más susceptibles a la desmineralización $(13,14)$. Los dientes parcialmente erupcionados no participan de la masticación, ya que no mantienen contacto con los antagonistas, acumulando en consecuencia una mayor cantidad de biofilm (1). A esto hay que agregar la presencia del capuchón gingival que también dificulta la limpieza y favorece la acumulación de biofilm y la aparición de lesiones cariosas en esta región (15).

La lesión cariosa oclusal se desarrolla desde las paredes laterales de la fisura, profundizándose en dirección de las estrías de Retzius para extenderse hasta el límite amelodentinario. En esas regiones, por sus características anatómicas, hay mayor dificultad para la remoción mecánica del biofilm; además, el flúor de las aguas de abastecimiento público y de las pastas dentales también tiene un papel limitado (6), haciendo fundamental la utilización de métodos precisos para el diagnóstico temprano y de tratamientos preventivos.

\section{Diagnóstico}

Por lo general, en estas superficies es difícil hacer un diagnóstico adecuado, especialmente cuando hay ausencia de una cavidad evidente (16). Las decisiones clínicas referidas a la caries dental son realizadas bajo condiciones inciertas, que 
involucran diversos tipos de juicio, y el diagnóstico debe reflejar la verdadera distinción inequívoca entre salud y enfermedad, con el propósito de seleccionar la forma más apropiada de intervención (1).

El diagnóstico de lesiones oclusales incipientes todavía es considerado problemático, debido a la compleja anatomía de las fosas y fisuras, asociada a la manifestación tardía de las cavidades y la frecuencia creciente de lesiones paralizadas, que obedecen a una modificación del patrón de desarrollo de la lesión (6).

El diagnóstico oclusal también se ve dificultado por el uso indiscriminado de flúor y por la aparición de la denominada "lesión oculta", debida al aumento de la capacidad de remineralización del esmalte que permite el desarrollo de una lesión en el fondo de la fisura que puede llegar hasta la dentina, manteniéndose intacta la superficie adamantina (17-19). Además, cabe la posibilidad de que haya un sellado biológico, con una masa amorfa de material orgánico, con pocas bacterias, y una mineralización progresiva que también puede enmascarar el diagnóstico (20).

Como consecuencia de estas características y particularidades, y de la dificultad del diagnóstico de la caries dental en superficies de fosas y fisuras, que han sido ampliamente discutidas en la literatura, han aparecido nuevos métodos de diagnóstico oclusal. Sin embargo, algunos de ellos, como la utilización de láser, transiluminación con fibra óptica, radiografía digital directa y resistencia eléctrica, son caros, de difícil acceso y no están disponibles para la práctica clínica diaria.

La transiluminación de fibra óptica se considera un método práctico y seguro para el diagnóstico de superficies oclusales, pero este apa- rato tiene un costo elevado y su precisión es cuestionable (21).

Los métodos de diagnóstico a base de láser, como DIAGNOdent , de la fabrica Kavo, son más sensibles y específicos que el método radiográfico convencional, principalmente en la detección de caries oculta. No obstante, ha demostrado tendencias hacia un diagnóstico falsopositivo, ya que no puede distinguir la lesión de caries de defectos del esmalte, acumulaciones de biofilm y cálculo dental $(22,23)$. Por esta razón, los exámenes clínico, visual y radiográfico constituyen todavía el método más común y disponible para la mayoría de los cirujano-dentistas.

Diversos autores se han preocupado de evaluar estos diferentes métodos de diagnóstico determinando su especificidad y sensibilidad, es decir, su real efectividad en la detección temprana de las lesiones de caries en la superficie oclusal.

Bader y Brown (7) sostienen que son muchas las divergencias entre los clínicos sobre la decisión de tratar o no una lesión de caries oclusal, y que son necesarios nuevos criterios y métodos para el diagnóstico de este tipo de lesiones.

De Assunção, Alves y De Oliveira (8) evaluaron la opinión de los cirujano-dentistas sobre el tratamiento y diagnóstico de lesiones de caries oclusal, observando grandes divergencias (68,5\%) en la detección de la enfermedad (diagnóstico visual) y una decisión basada en una filosofía de tratamiento restaurador. Destacaron que el diagnóstico falso-positivo lleva a un tratamiento innecesario, que puede traer secuelas negativas por la remoción de tejido dental sano, un mayor costo económico y la utilización innecesaria de recursos humanos.

Tradicionalmente, aunque no se considera el más efectivo, es muy frecuente la utilización del método táctil-visual con explorador y espejo bucal (2). Lussi (6) demostró que el método visual y el método con sonda fueron los menos sensibles. Sin embargo, cuando se agregó la radiografía interproximal a estos métodos, se convirtieron en los más precisos de todos los estudiados.

Para la detección de lesiones de caries, Pereira y Moreira (15) compararon la eficacia y confianza de tres tipos de exámenes: 1) examen clínico con explorador, espejo bucal y luz natural, 2) explorador, espejo y luz artificial y 3) uso de medios auxiliares, como radiografías interproximales y fibra óptica. Los resultados indicaron que los métodos auxiliares aumentaron las medidas del índice CPOD en $35,7 \%$ y $15 \%$, respectivamente, en comparación con las que se obtuvieron con los exámenes 1 y 2 . Un estudio sobre la importancia del método visual y radiográfico demostró que solamente un tercio de las lesiones oclusales en dentina fueron detectadas clínicamente y que las restantes se descubrieron sólo mediante radiografías interproximales (20).

Milicich (24)también demostró que el explorador y el espejo bucal tienen apenas el 25\% de precisión cuando el examen se realiza en condiciones ideales, es decir, en dientes limpios, secos y con buena iluminación. Poorterman et al. (17), en un estudio semejante, demostraron que solamente la quinta parte de las lesiones en dentina se detectan clínicamente.

La importancia de las radiografías como examen auxiliar de diagnóstico también ha sido destacada por Weerheijim (25), debido a que permite detectar lesiones de caries oculta, que se caracterizan por la presencia de esmalte sano o mínima-mente afectado, con compromiso de dentina. Esto explica lo difícil 
que es identificar estas lesiones sólo con el examen visual. De otro lado, en un estudio clínico, Poorterman et al. (17) observaron que la prevalencia clínica de caries en primeros y segundos molares fue muy subestimada, al compararla con la prevalencia radiográfica.

Pereira et al. (16) compararon la precisión de los métodos táctiles y visuales con el examen de electromicroscopía en el diagnóstico de lesiones de caries en fosas y fisuras, concluyendo que el sondeo clínico todavía es un método muy usado, el examen visual sin explorador parece no influenciar en la proporción de aciertos de diagnóstico y que el diagnóstico en superficies oclusales es difícil.

Meneghim et al. (26) compararon diversos métodos de diagnóstico de caries dental y concluyeron que el examen clínico visual en dientes limpios, secos y bien iluminados mejora el diagnóstico de la lesión y que, cuando se asocia con el examen radiográfico, la precisión aumenta en 4\%. Mestriner et al. (27), después de evaluar distintos métodos de diagnóstico, concluyeron que los métodos tradicionales, la inspección visual y las radiografías, son efectivos y complementarios en la detección de caries.

A pesar de haber confirmado la importancia del examen radiográfico convencional, Wenzel (28) sostiene que este método da una visión bidimen-sional de una estructura que tiene tres dimensiones y, por lo tanto, presenta limitaciones. Además, hay dificultad para estandarizar la toma de radiografías, agregando que la evolución de los sistemas de radiografías digitales traerá beneficios adicionales, como la estandarización del examen, economía de tiempo y reducción de la radiación, tanto para el profesional como para el pacien- te. Por lo tanto, como no hay diferencias en la precisión de las radiografías digital y convencional, no se conoce con exactitud la relación entre costo y beneficio a la hora de escoger una de ellas $(28,29)$.

Un estudio que evaluó las diferentes estrategias actuales de diagnóstico a base de calidad de luz fluorescente inducida(QFL), DIAGNOdent y monitor electrónico de caries (ECM), concluyó que no existen evidencias suficientes para que sean indicadas como sustitutos de las técnicas de diagnóstico convencionales, y que el examen radiográfico interproximal todavía es el método más utilizado, sabiendo que su eficacia es mayor cuando la lesión ya ha alcanzado la dentina (30).

Ribeiro et al. (19) mencionan las ventajas de asociar métodos modernos de diagnóstico, como el sistema QFL, para evaluar la presencia de lesiones cariosas, resaltando que este aparato posee un alto potencial de diagnóstico.

Por las particularidades y limitaciones ya discutidas de todos los métodos de diagnóstico disponibles, el cirujano-dentista muchas veces necesita hacer una invasión mínima para establecer un diagnóstico preciso, mediante un procedimiento llamado diagnóstico invasivo $(4,31)$.

El examen radiográfico debe ser considerado de fundamental importancia, principalmente por ser un método auxiliar altamente efectivo y al alcance del clínico, y puede ser conclusivo en la mayoría de las veces en la detección de lesiones cariosas incipientes en la superficie oclusal.

En conclusión, el profesional debe considerar que frente tantas opciones el método clínico visual y radiográfico es el más común y de fácil acceso y que, cuando se realiza en condiciones ideales, es efectivo en la mayoría de las situaciones de la práctica clínica diaria. Cuando sea necesario, el dentista clínico deberá realizar el diagnóstico invasivo, como una alternativa segura y de carácter preventivo.

Los cambios en el patrón del desarrollo de la caries dental y la importancia de la odontología preventiva convierten los métodos de diagnóstico en una herramienta fundamental para que el clínico pueda intervenir precozmente, de forma correcta, las lesiones de caries dental incipiente en superficies oclusales.

\section{Referencias bibliográficas}

1. Fejerskov O, Kidd E. Cárie Dentária: A doença e seu tratamento clínico. São Paulo: Livraria Editora Santos; 2005.

2. Rock WP, Gordon PH, Bradnock G. Caries experience in West Midland school children following fluoridation of Birmingham water in 1964. Caries of first permanent molars. Br Dent J. 1981; 150(10): 269-73.

3. Dirks OB. The benefits of water fluoridation. Caries Res. 1974; 8(0):suppl:2-15.

4. Meiers JC, Jensen ME. Management of the questionable carious fissure : invasive vs noninvasive techniques. J Am Dent Assoc. 1984; 108(1):64-8.

5. Buonocore MG. A simple method of increasing the adhesion of acrylic filling materials to enamel surfaces. J Dent Res. 1955; 34(6):849-53.

6. Lussi A. Comparison of different methods for the diagnosis of fissure caries without cavitation. Caries Res. 1993; 27(5):409-16.

7. Bader JD, Brown JP. Dilemmas in caries diagnosis. J Am Dent Assoc. 1993; 124(6):48-50.

8. De Assunção IV, Alves MDoSCF, De Oliveira MAF. 
Cárie oclusal: uma avaliação clínica e de microdureza do esmalte. RPG. 2003; 10(2):120-30.

9. Navarro MFL, Franco EB, Mondelli J, Pereira JC, Martins LRM, Pinheiro CE. Remineralization of enamel white spotlesions. Estomat Cult. 1985; 15(4): 41-6.

10. Carvalho JC, Ekstrand KR, Thylstrup A. Dental plaque and caries on occlusal surfaces of first permanent molars in relation to stage of eruption. J Dent Res. 1989; 68(5):773-9.

11. Carvalho J, Maltz M. Tratamento da doença cárie. En: ABOPREV. Promoção de saúde bucal. São Paulo: Artes médicas; 1997:95-112.

12. Ekstrand KR, Nielsen LA, Carvalho JC, Thylstrup A. Dental plaque and caries on permanent first molar occlusal surfaces in relation to sagittal occlusion. Scand J Dent Res. 1993; 101(1):9-15.

13. Kotsanos N, Darling AI. Influence of posteruptive age of enamel on its susceptibility to artificial caries. Caries Res. 1991; 25(4):241-50.

14. Geller, D. Avaliação da desmineralização produzida por desafio cariogênico in situ em esmalte dentário com deferentes idades póseruptivas. [Dissertação]. Bauru (SP): Faculdade de Odontologia de Bauru. Universidade de São Paulo; 2007.

15. Pereira AC, Moreira BW. Diagnóstico da cárie dentária: estudo comparativo de diferentes métodos de exame utilizados em odontologia. RGO. 1995; 43(3): 127-31.

16. Pereira CRS, et al. Avaliação da presição dos métodos visual e radiográfico no diagnóstico de cárie de cicatrículas e fissuras. JBP. 2001; 4(19):197-202.

17.Poorterman JH, Weerheijm KL, Groen HJ, Kalsbeek H. Clinical and radiographic judgement of occlusal caries in adolescents. Eur J Oral Sci. 2000; 108(2):93-8.

18. Prakki A, Campos BB, Regalado, D, Bresciani E, Capelozza AL. Cárie oculta : uma visão atual. Salusvita. 2002; 21(1):67-76.

19. Ribeiro A, Rousseau C, Girkin J, Hall A, Strang R, John Whitters C, Creanor S, Gomes AS. A preliminary investigation of a spectroscopic technique for the diagnosis of natural caries lesions. J Dent. 2005; 33(1):73-8.

20.Ricketts D, Kidd E, Weerheijm $\mathrm{K}$, de Soet $\mathrm{H}$. Hidden caries: what is it? Does it exist? Does it matter? Int Dent J. 1997; 47(5): 259-65.

21.Cortes DF, Ekstrand KR, EliasBoneta AR, Ellwood RP. An in vitro comparison of the ability of fibre-optic transillumination, visual inspection and radiographs to detect occlusal caries and evaluate lesion depth. Caries Res. 2000; 34(6):443-7.

22.Lussi A, Imwinkelried S, Pitts N, Longbottom C, Reich E. Performance and reproducibility of a laser fluorescence system for detection of occlusal caries in vitro. Caries Res. 1999; 33(4): 261-6.

23. Shi XQ, Welander U, AngmarMansson B. Occlusal caries detection with $\mathrm{KaVo}$ DIAGNOdent and radiography: an in vitro comparison. Caries Res. 2000; 34(2):151-8.

24. Milicich G. Clinical applications of new advances in occlusal caries diagnosis. N Z Dent J. 2000;
96(423):23-6.

25. Weerheijm KL. Occlusal 'hidden caries'. Dent Update. 1997; 24(5):182-4.

26. Meneghim Mde C, Assaf AV, Zanin L, Kozlowski FC, Pereira AC, Ambrosano GM. Comparison of diagnostic methods for dental caries. J Dent Child. 2003; 70(2):115-9.

27. Mestriner SF, Vinha D, Mestriner Jr W. Comparison of different methods for the occlusal dentine caries diagnosis. J Appl Oral Sci. 2005; 13(1): 28-34.

28. Wenzel A. Bitewing and digital bitewing radiography for detection of caries lesions. J Dent Res. 2004; 83(Spec No C):C72-5.

29. Hintze H, Wenzel A, Jones C. In vitro comparison of $\mathrm{D}$ - and $\mathrm{E}$ speed film radiography, RVG, and visualix digital radiography for the detection of enamel approximal and dentinal occlusal caries lesions. Caries Res. 1994; 28(5): 363-7.

30.Tranaeus S, Shi XQ, AngmarMansson B. Caries risk assessment: methods available to clinicians for caries detection. Community Dent Oral Epidemiol. 2005; 33(4):265-73.

31.De Craene GP, Martens C, Dermaut R. The invasive pitand-fissure sealing technique in pediatric dentistry: an SEM study of a preventive restoration. ASDC J Dent Child. 1988; 55(1): 34-42.

32.Pérez A, Quenta Silva E, Cabrera Matta A, Cárdenas Campos D, Lazo Navarro R, Lagravère Vich M. Caries dental en dientes deciduos y permanentes jóvenes: diagnóstico y tratamiento conservador. Lima: Universidad Peruana Cayetano Heredia; 2004. 